\title{
Una aproximación al concepto de impacto como emergente triádico: un insumo para el análisis de la convivencia ciudadana
}

\author{
Celio Arnulfo Téllez-Aroca ${ }^{\star}$, Blanca Lilia Castañeda-Barón ${ }^{\star *}$, \\ Claudia Alexandra Guzmán-Pinzón ${ }^{\star * *}$, Jesús Aníbal Gantiva-Rocha ${ }^{\star * * *}$
}

* Maestría(c) en Educación, Universidad Cooperativa de Colombia. Docente de Ciencias Sociales, sED, Bogotá, Colombia. Correo electrónico: arnulfotel@yahoo.com

* Maestría(c) en Educación, Universidad Cooperativa de Colombia. Docente de Ciencias Sociales, SED, Bogotá, Colombia. Correo electrónico: liliacas2004@yahoo.com

*** Maestría(c) en Educación, Universidad Cooperativa de Colombia. Docente de Ciencias Naturales, Colegio Cervantes, Bogotá, Colombia. Correo electrónico: claudiaguzmanpinzon@hotmail.com

${ }^{* * * *}$ Maestría(c) en Educación, Universidad Cooperativa de Colombia. Docente de Ciencias Naturales, SED, Bogotá, Colombia. Correo electrónico:

jesus.anibal@yahoo.com

Recibido: 15 de agosto del 2014

Aprobado: 28 de octubre del 2014

Cómo citar este artículo: Téllez-Aroca, Celio, Blanca Castañeda-Barón, Claudia Guzmán-Pinzón y Jesús Gantiva-Rocha. "Una aproximación al concepto de impacto como emergente triádico: un insumo para el análisis de la convivencia ciudadana". Rastros Rostros 16.30 (2014): 129-133. Impreso. doi: http://dx.doi.org/10.16925/ra.v16i30.821

\begin{abstract}
Resumen
En el artículo se presenta una propuesta que amplía los componentes e implicaciones del concepto de impacto, para permitirle nuevas dimensiones y rangos tanto de caracterización como de medición, además de múltiples posibilidades de interpretación. Se toma como punto de partida el rastreo del concepto impactus en estudios de diversos centros académicos, luego se infieren aspectos constitutivos para contrastarlos y complementarlos desde la perspectiva de la cibernética social. Este ejercicio permitió plantear la existencia (en los referentes consultados), de tendencias unívocas, homogenizantes y excluyentes de los tres procesos interdependientes de los seres humanos (el actuar, el sentir y el pensar), que contrastan con el impacto como emergente. El reto por darle sentido desde la proporcionalidad de las potencialidades del ser humano es generar el descubrimiento de nuevas maneras y opciones de acercamiento al mundo de las aristas de influencia de los programas académicos universitarios de posgrado en educación, de los individuos, la sociedad y el mundo.
\end{abstract}

Palabras clave: cibernética social, convivencia, impacto, Maestría en Educación, ontología del lenguaje.

Examining the Concept of Impact as a Tripartite Emergence: A Contribution for the Analysis of Citizen Coexistence

\begin{abstract}
This article presents a proposal to widen the components and implications of the concept of "impact", to reveal new aspects and scope for both its characterization and measurement, as well as offering various options for interpreting it. As a starting point, the concept of impactus is examined in studies from various academic centers, and its aspects are then inferred in order to contrast and complement them from a sociocybernetics perspective. Through this exercise, it was possible to see the existence (in the reference works consulted) of clear, homogenizing and exclusive trends in the three independent processes of human beings (acting, feeling and acting), that contrast with impact as an emergence. The challenge in making sense of this from the proportionality of human potential is to discover new ways of approaching the world of signs of influence in post-graduate university programs, individuals, society, and the world.
\end{abstract}

Keywords: sociocybernetics, coexistence, impact, Master of Education, ontology of language.

Uma aproximação ao conceito de impacto como emergente tríadico: um insumo para a análise da convivência cidadã

\section{Resumo}

Este artigo apresenta uma proposta que amplia os componentes e implicações do conceito de impacto, para permitir novas dimensões e categorias tanto de caracterização quanto de medição, além de múltiplas possibilidades de interpretação. Toma-se como ponto de partida o rastreamento do conceito impactus em estudos de diversos centros acadêmicos, logo se inferem aspectos constitutivos para contrastá-los e complementá-los a partir da perspectiva da cibernética social. Esse exercício permitiu explicar a existência (nos referentes consultados), de tendências unívocas, homogeneizantes e excludentes dos três processos interdependentes dos seres humanos (o agir, o sentir e o pensar), que contrastam com o impacto como emergente. O desafio para dar sentido a partir da proporcionalidade das potencialidades do ser humano é gerar o descobrimento de novas maneiras e opçães de aproximação ao mundo das aristas de influência dos programas acadêmicos universitários de pósgraduação em educação, dos indivíduos, da sociedade e do mundo.

Palavras-chave: cibernética social, convivência, impacto, Mestrado em Educação, ontologia da linguagem. 


\section{Introducción}

La categoría de impacto puede ser repensada y el lenguaje le permite recrear nuevos mundos de sentido. A partir del análisis sistemático realizado, no sólo sobre el significado o las implicaciones del concepto de impacto, sino de los ambientes y contextos en los que tiene su aparición, los referentes le permiten al ser humano distintas apreciaciones sobre la realidad. De igual manera, la intencionalidad le podrá permitir reconocer los eslabones de la producción cultural sobre los cuales forjar nuevas posibilidades de construcción proyectadas en etapas venideras. En tal sentido, la Universidad Centroamericana, citada por Aguilar (53), considera la existencia de dos grandes ámbitos de influencia que experimentan los estudiantes a su paso por la universidad: el interno y el externo. En el primero, se encuentran los cambios experimentados por los sujetos y, en el segundo, están los aportes y transferencias que realizan en su región o comunidad. Elejalde y Várcarcel, citados por Couturejuzón, Rubén y González (6), catalogan el impacto como uno de los atributos que debe contemplarse en el proceso de evaluación de las instituciones y (no sólo) a docentes.

Así, los mundos de sentido dados al concepto de impacto por parte de Aguilar (54) y Couturejuzón, Rubén y González (25) propician la cocreación de micro-, meso- y macromundos a partir del lenguaje, que pueden ser complementados por los vocablos, categorías, macroestructuras, propuestas, subsistemas, vidogramas (la vida reflejada en gráficos), de la cibernética social.

De este modo, los vectores que acompañan el concepto de impacto (calidad, eficiencia, transferencia, aportes, entre otros), pueden ser integrados desde la proporcionalidad biológica, antropológica, cultural y cosmológica del ser humano.

Es entonces cuando se ve la necesidad de hablar no deimpacto sino de impactos, más allá de los planosinternos y externos, de categorías espacio-temporales y de la interdependencia, interafectación e interrelación de unos con otros, para acrecentar el sentido tanto del todo como de las partes. El diálogo y el encuentro entre los impactos pueden generar nuevos episodios de engrandecimiento de su caracterización, que convoquen a realizar ajustes y reingenierías en los procesos de diseño e implementación de los programas académicos con carácter de universidad-universalidad.

Quizás la única razón para llevar a cabo el estudio es lo que se podría llamar la caja de herramientas colectiva. Así, como otros plantearon elementos para recabar, comprender y aplicar aristas del impacto, el grupo de investigación se siente convocado a agregar algo a ese arsenal que pueda ser en algún grado útil a otros.

Son varios los condicionantes que inciden en la credibilidad del artículo. Primero, la pseudouniversalidad, ya que los artículos muestra de estudio son escasos. A esto se le debe sumar la carencia de canales formales de comunicación de la Universidad Cooperativa de Colombia con pares de otros idiomas y niveles de desarrollo científico, tecnológico y cultural. El segundo, el más decisivo, es la poca disciplina y rigurosidad para concebir un artículo científico en una veintena de días, entre ellos, varios festivos y religiosos.

En el desarrollo de la investigación del equipo titulada "Análisis del impacto de la gestión de la investigación realizada por los egresados en la maestría en educación de la Universidad Cooperativa de Colombia", se considera que un elemento a incorporar en la caracterización del impacto de programas de maestría en educación es el de las competencias socio-emocionales. En el mismo sentido que Velandia y Betancourt escriben: "Es necesario que en los procesos de formación en la universidad se relacionen la vida emocional, la cognición y comportamiento de manera sinérgica. Se integre lo académico con las competencias socioemocionales" (9). Esto se constituye como una propuesta frente al problema que describen del siguiente modo:

Nos hemos centrado en las competencias intelectuales dejando en un segundo plano la educación de las emociones, la afectividad y los sentimientos, dando lugar a una desproporcionalidad entre lo cognitivo, lo emocional y procedimental en los contenidos curriculares.(2)

En concordancia con lo anterior, vale la pena mencionar lo que plantea Echeverría (citado por Velandia "Somos seres lingüísticos" 4):

Cada planteamiento hecho por un observador nos habla del tipo de observador que ese observador considera que es ... hagamos lo que hagamos, digamos lo que digamos, siempre se revela en ello una cierta comprensión de lo que es posible para los seres humanos.

Con esto se reitera que, a partir de la interpretación de la realidad y del ser humano, hecha desde la cibernética social, se pretende abordar el mundo y el concepto del impacto encarnado y expresado por otras instituciones académicas, tanto de orden nacional como internacional. 
Es evidente que los procesos de formación académica en los programas de maestría en educación se dan en las interacciones con otros (pares académicos, docentes, directivos, investigadores, administrativos, colaboradores), que en la cibernética social están asociadas al factor emocional. En este sentido, Velandia considera que "el docente debe aprender a educarse emocionalmente; es decir, a asumirse en su ser emocional y a vivenciar las emociones como parte fundamental de su proceso pedagógico y de su ejercicio didáctico, dado que las emociones son los motores de la acción humana" ("Comunicación y educación emocional" 1). Si bien existe la tendencia a asociar programas académicos con el plano cognitivo, en este caso estudios de maestría, Velandia considera que "más que seres racionales somos seres emocionales ya que en nuestras vidas no hay ningún momento libre de emociones, de ahí la importancia de centrarnos en conocerlas ..." ("Cambio social” 2). En consecuencia, es pertinente configurar indicadores que permitan indagar su existencia en los egresados de maestrías, es decir, hacer visible cómo la universidad logra impactar en el mundo emocional de los egresados (algunas de las acciones de los egresados son la expresión de la incidencia de los programas académicos y la manifestación de las emociones), que se constituye en el insumo del actuar.

Goijberg considera que "toda nuestra cultura y educación nos ha hecho desarrollar sólo la inteligencia racional y no la emocional y por eso las difíciles relaciones entre personas, entre pareja, laborales, maestroalumno, etcétera" (citado por Velandia "Cambio social" 2). En tal sentido, el grupo de investigación se encuentra en la elaboración de unas posibles pautas e interrogantes que permitan el rastreamiento de la influencia de los programas académicos en el ámbito profesional de los egresados dela Maestría en Educación, considerando que, para la Universidad Cooperativa de Colombia, existe la figura tanto de egresado como de egresado graduado (Universidad Cooperativa de Colombia 17).

Ahora bien, el planteamiento de indicadores de impacto de orden emocional en los egresados de maestrías en educación se apoya en Velandia ("Cambio social" 2), que concluye: "Goleman ha jugado un papel determinante en el creciente reconocimiento que las emociones tienen actualmente e incluso, en las derivaciones que sus estudios y publicaciones han logrado".

Teniendo como marco referencial la teoría de sistemas, se puede observar cómo hacia el interior de las universidades los subsistemas no se agotan en sí mismos hacia el exterior. Igualmente, se puede ver que se encuentran interactuando con la sociedad en una permanente dinámica que permite visibilizar aportes al mejoramiento del macrosistema, al suyo y al de sus subsistemas.

Velandia concibe el cambio social como "la emergencia económica, política o cultural que surge del conflicto que se genera por la interrelación, interafectación e interdependencia entre los agentes de cambio, sectores, instituciones y el medio" ("Comunicación y educación emocional" 3). Por esta razón, dentro del espectro emergente de los profesionales, se hacen necesarios los aportes a la emocionalidad desde la universidad, y que vea en estos sujetos que experimenten y propendan hacia prácticas sociales proporcionales y sistémicas. En concordancia con lo anterior, se puede colegir que no es suficiente sólo la vivencia y expresión del mundo de las emociones, acciones y pensamientos de los individuos, sino que se necesita para la materialización de los proyectos personales de los otros y de los ambientes en los que sea viable la convivencia.

La convivencia reconoce la coexistencia simultánea de personas, identidades (tanto como sujetos de derechos, como de ciudadanos), mismidades, alteridades y la consciencia de la liminaridad, como lo expresa Velandia: "Es igualmente esencial la conciencia de los límites relacionales intelectuales, emocionales y comportamentales existentes con los otros, las otras y el medio - liminaridad-, ya que ello afecta nuestros versos, emociones y experiencias" ("Sujetos de acción de la educación para la convivencia” 13).

\section{Elementos de discusión}

a) Según Velandia, "el conocimiento es lo que queda de la interacción, interafectación e interdependencia de sus experiencias, emociones y saberes intelectuales." ("Somos seres linguísticos" 12). La categoría de impacto, constituida como la emergencia resultante de la interacción, interafectación e interdependencia de las experiencias-emociones y saberes intelectuales del grupo que cursa la Maestría en Educación, desarrolla el proyecto de investigación e incorpora al universo de sentido de impacto o, por lo menos, desde lo que plantea tanto Couturejuzón, Rubén y González como Aguilar, ámbitos holísticos. Si bien debe reconocerse como íntegral y como un subsistema más, no debe considerarse a sí misma como perfecta y no susceptible de mejoramiento, porque entraría a consolidarse como unívoca monáica. 
b) Según Velandia, "el lenguaje no sólo permite describir realidades, también las crea, genera ser ..." (“Somos seres linguísticos” 5). De este modo, el grupo de investigación, más allá de describir los referentes existentes sobre el impacto de las maestrías en educación y las categorías de calidad a la luz de Cardoso-Espinosa y Cerecedo-Mercado (pertinencia, trascendencia, equidad, eficacia y eficiencia) y del documento del Consejo Nacional de Acreditación, induce la posibilidad de generar nuevos instrumentos para el rastreamiento de acciones, emociones y pensamientos de egresados a partir de los cuales hacer mejoras en los programas. ¿Qué proceso y qué validez debe contener la generación de sentidos a partir de emergencias particulares, para aspirar a tener un criterio de universalidad, considerando que esta ha de ser consustancial al magma constitutivo de las universidades?

c) Apoyando la discusión, se tiene la apreciación: "Toda nuestra realidad humana es social y somos individuos, personas, sólo en cuanto somos seres sociales en el lenguaje ..." (Maturana citado por Velandia "Somos seres lingüísticos" 5). El lenguaje, la creación de palabras y los sentidos, desde lo emergente, procuran la coexistencia social, siendo una de sus expresiones la convivencia ciudadana. No obstante, más allá de la interafectación sujeto, persona y ciudadano, es imprescindible apuntalar actuares, sentires y pensares de la convivencia en el plano orbital, mientras que el tópico de ciudadano se circunscribe a las realidades de personas dentro de diversos territorios-estados-naciones, pero existen vínculos de intercomunicación entre sujetos de distintas ciudadanías, de donde emergen nuevas manifestaciones operativas, emocionales y lógicas.

\section{Conclusiones}

Las interacciones de referentes, expresiones, mundos emocionales y lógicas de pensamiento, aunadas a la posibilidad emergente del ser humano fundada en el lenguaje, permiten la explosión de innumerables vocablos, constructos, prácticas e intercambios. Sin embargo, se requiere el reconocimiento de lazos, rutas, vasos comunicantes, no sólo para interrelacionarlos, sino para propender hacia un lenguaje universal a partir del cual adicionar, purificar y concebir emergencias aptas para la coexistencia, supervivencia y trascendencia de la especie humana. No es pertinente evaluar la validez de todas las emergencias suscitadas por los humanos: se deberán legitimar aquellas que provoquen el aseguramiento de la convivencia y de la calidad de vida de los homínidos actuantes, sentientes y pensantes y de otras formas de vida. Es así que la categoría de impacto desde la atmósfera de la cibernética social podrá nombrar, apalabrar, reconocer realidades que por presencia o ausencia inciden no sólo en el entorno, sino en el medio y el mundo. Al agregar una nueva herramienta al taller de los programas de maestría para percibir el impacto, se transitará por una serie de momentos, $y$, si demuestra su utilidad, profundidad y relatividad, podrá iniciar una nueva fase, adentrarse en otras circunstancias espacio-temporales y vivenciales. En este sentido se plantea el impacto como emergente de los tres procesos interdependientes de los seres humanos (el actuar, el sentir y el pensar) mostrado en la figura 1.

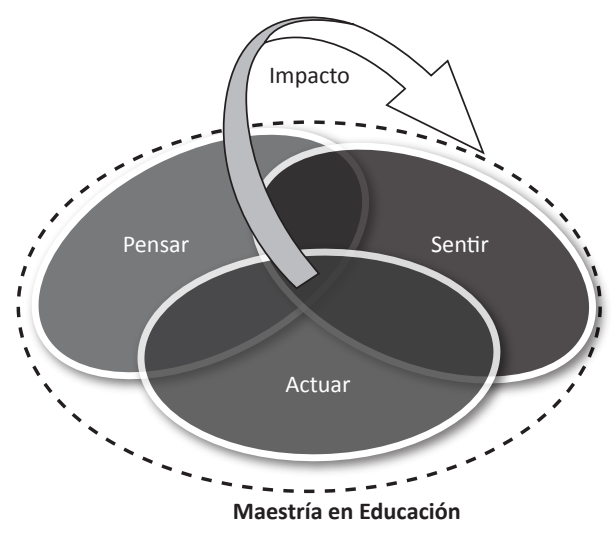

Figura 1. Impacto como emergencia de las tres potencialidades, Universidad Cooperativa de Colombia, 2014. Elaboración propia.

\section{Recomendaciones}

La primera es poner en diálogo con otros académi$\cos$ (conocedores, investigadores, mentores, asesores), dentro de emergencias alternativas (sistémicas, holísticas, emocionales, tríadicas), los hallazgos aquí presentados para consolidar un acumulado que permita fortalecer y hacer una propuesta a los diversos mundos con el fin de hablar de impacto proporcional. La segunda es incursionar en el mundo de la convivencia más allá de sujeto, persona y ciudadano. Finalmente, promover los diálogos y encuentros entre visiones, enfoques, paradigmas, con la pretensión de reconocer y valorar los aportes de los más disímiles arquetipos que han labrado las actuales condiciones de convivencia. 


\section{Referencias}

Aguilar, Miguel Ángel. El impacto de la carrera de economía de la BUAP en el mercado laboral: la visión de los egresados de la generación 1995-2000. Biblioteca Virtual de Derecho, Economía y Ciencias Sociales. 2009. Web.

Cardoso-Espinosa, Edgar, y María Trinidad Cerecedo-Mercado. "Propuesta de indicadores para evaluar la calidad de un programa de posgrado en Educación”. Revista electrónica de investigación educativa 13.2 (2011): 6882. Impreso.

Consejo Nacional de Acreditación. El sistema de mejoramiento continuo del Consejo Nacional de Acreditación (CNA). Bogotá: Consejo Nacional de Acreditación, 2011.

Couturejuzón, Lourdes, Mercedes Rubén y Nery González. "Impacto de la Maestría Informática en Salud en la esfera intelectual del egresado. Instituto Superior de Ciencias Médicas de la Habana. 1997-2002". Revista Cubana de Informática Médica. 2004. Web

Universidad Cooperativa de Colombia. Reglamento Estudiantil. (2014). Web.

Velandia, Manuel Antonio. "Cambio social". Módulo de Educación para la Convivencia. Universidad Cooperativa de Colombia. España, Madrid. 2011. Impreso.

. “Comunicación y educación emocional". 2011. Web.

- "Somos seres linguísticos: aportes desde la ontología del lenguaje”. 2013.Web.

. "Sujetos de acción de la educación para la convivencia”. 2013. Web.

Velandia, Manuel Antonio y Stella Betancourt. Competencias socioemocionales. 2012. Web. 